\title{
A phase 1 dose-escalation study of the oral histone deacetylase inhibitor abexinostat in combination with standard hypofractionated radiotherapy in advanced solid tumors
}

\author{
Eric Deutsch ${ }^{1,2,3}$, Elizabeth Cohen-Jonathan Moyal ${ }^{4}$, Vanesa Gregorc 5 , Paolo Andrea \\ Zucali, Jean Menard ${ }^{7}$, Jean-Charles Soria ${ }^{8}$, Ioana Kloos ${ }^{9}$, Jeff Hsu $^{10}$, Ying Luan ${ }^{10}$, \\ Emily Liu ${ }^{10}$, Remus Vezan ${ }^{10}$, Thorsten Graef ${ }^{10}$ and Sofia Rivera ${ }^{1,2,3}$ \\ ${ }^{1}$ Department of Radiation Oncology, Gustave-Roussy Cancer Campus, Villejuif, France \\ ${ }^{2}$ INSERM 1030 Molecular Radiotherapy, Villejuif, France \\ ${ }^{3}$ Faculté de Médecine, Université Paris-Sud, Université Paris-Saclay, Le Kremlin-Bicêtre, France \\ ${ }^{4}$ Department of Radiation Oncology, Institut Claudius Regaud, Toulouse, France \\ ${ }^{5}$ Department of Medical Oncology, Istituto di Ricovero e Cura a Carattere Scientifico, Ospedale San Raffaele, Milan, Italy \\ ${ }^{6}$ Department of Medical Oncology and Haematology, Humanitas Cancer Center, IRCCS, Rozzano, Italy \\ ${ }^{7}$ Department of Radiation Oncology, Hopital Saint-Louis, Paris, France \\ ${ }^{8}$ DITEP (Département d'Innovations Thérapeutiques et Essais Précoces), Gustave Roussy Cancer Campus, Villejuif, France \\ ${ }^{9}$ Institut de Recherches Internationales Servier, Clinical Pharmacokinetics, Suresnes, France \\ ${ }^{10}$ Pharmacyclics LLC, an AbbVie Company, Sunnyvale, CA, USA \\ Correspondence to: Eric Deutsch, email: eric.deutsch@gustaveroussy.fr \\ Keywords: abexinostat, histone deacetylase inhibitor, radiotherapy, solid tumors, brain lesions \\ Received: July 28,2016 Accepted: November 14, 2016 Published: December 24, 2016 \\ Copyright: Deutsch et al. This is an open-access article distributed under the terms of the Creative Commons Attribution License \\ 3.0 (CC BY 3.0), which permits unrestricted use, distribution, and reproduction in any medium, provided the original author and \\ source are credited.
}

\section{ABSTRACT}

Current treatments for advanced solid tumors tend to be only palliative. Although radiotherapy is administered with a curative intent, radioresistance and dose-limiting toxicities pose limitations to treatment. Abexinostat, an oral pan-histone deacetylase inhibitor, demonstrated enhanced sensitivity to radiation in various solid tumor cell lines. We conducted an exploratory, phase 1, dose-escalation study of abexinostat in combination with standard hypofractionated radiotherapy in patients with advanced solid tumors treated in a palliative setting. Among 58 treated patients, the median age was 61.5 years (range, 20-82); $47 \%$ of the patients had M1 stage disease, and $95 \%$ had received previous chemotherapy alone or chemotherapy in combination with surgery and/or radiotherapy. The recommended phase 2 dose was determined to be $90 \mathrm{mg} / \mathrm{m}^{2}(140 \mathrm{mg})$. Of the 51 patients evaluable for response, best overall response was $8 \%$ ( 1 complete response [CR], 3 partial responses [PRs]), and best loco-regional response was $12 \%$ ( 1 CR and 5 PRs) at a median follow-up of 16 weeks. Of note, patients with target or non-target brain lesions showed encouraging responses, with 1 patient achieving a best loco-regional response of CR. Treatment-emergent grade $\geq 3$ adverse events (AEs) were few, with most common being thrombocytopenia $(17 \%)$, lymphopenia (12\%), and hypokalemia (7\%). Six patients (10\%) discontinued treatment due to AEs. No grade $\geq 3$ prolongation of the QTc interval was observed, with no treatment discontinuations due to this AE. Oral abexinostat combined with radiotherapy was well tolerated in patients with advanced solid tumors. The combination may have potential for treatment of patients with brain lesions. 


\section{INTRODUCTION}

Epigenetic changes such as DNA methylation and post-translational histone acetylation play a critical role in the development of cancer due to their ability to alter the accessibility of transcription factors to DNA and chromatin structure [1]. Histone deacetylases (HDACs) enzymatically remove acetyl groups from histones and thus serve as key regulators of gene expression. Tumorigenesis is linked to aberrant activity of HDACs such as deacetylation of the tumor suppressor gene $p 53$, leading to its decreased transcription [2], and HDAC-mediated upregulation of oncogenes such as BCL2 [3]. The US Food and Drug Administration has approved the HDAC inhibitors (HDACIs) vorinostat and romidepsin for the treatment of cutaneous T-cell lymphoma, and romidepsin and belinostat are approved for the treatment of peripheral T-cell lymphoma. Panobinostat in combination with bortezomib and dexamethasone has been approved for the treatment of multiple myeloma. These agents have also been evaluated as single agents in a variety of solid tumors but have shown limited or no activity [4-11]. Due to the broad specificity and moderate activity of the existing HDACIs observed in these studies, a combinatorial approach has been suggested.

Abexinostat is an oral, broad-spectrum, phenyl hydroxamic acid-based pan-HDAC inhibitor that has demonstrated antitumor activity as a single agent in neuroblastoma cell lines [12], as well as in combination with bortezomib in mouse xenograft models [12]. Additionally, abexinostat as a single agent and in combination with chemotherapy has demonstrated significant antiproliferative activity in human soft tissue sarcoma models [13]. Abexinostat-induced apoptosis is known to occur through caspase- 8 and the Fas-associated death domain and is associated with a prominent increase in reactive oxygen species [14].

In recent years, HDACIs are emerging as promising radiosensitizing agents that play a critical role in cellular processes, such as cell growth and differentiation, apoptosis, and DNA repair [15]. Pre-treatment with abexinostat enhanced sensitivity to radiation in prostate, colon, lung, and cervical tumor cell lines [16, 17], supporting the clinical role for HDACIs in radiosensitization. HDAC inhibition by abexinostat led to a decrease in the number of cells able to form colonies after irradiation compared with radiation alone. Pre-treatment of colon tumor cells with abexinostat before irradiation induced a strong inhibition of RAD51-containing subnuclear repair foci formation, suggesting that abexinostat may act, in part, by inhibiting DNA repair [17]. Based on the pre-clinical studies showing activity of abexinostat in solid tumor models and enhanced radiosensitization by abexinostat in solid tumor cell lines, an exploratory phase 1 , dose escalation study of abexinostat in combination with standard hypofractionated radiotherapy was conducted in patients with advanced solid tumors treated in a palliative setting.

\section{RESULTS}

\section{Patients}

A total of 62 patients were enrolled in the study between September 2, 2010, and March 25, 2015. Fifty-eight patients received at least 1 dose of the study treatment, of whom 33 patients were treated in schedule 1 and 25 patients in schedule 2. Four patients were withdrawn from the study ( 3 due to AEs and 1 due to protocol deviation). The number of patients enrolled by schedule and group (supra- and subdiaphragmatic) is shown in Supplementary Table 1. Baseline characteristics of all treated patients are summarized in Table 1. The median age was 61.5 years (range, 20-82), with $38 \%$ of patients aged 65 years. The most common primary diagnoses were breast cancer (36\%), followed by lung cancer $(24 \%)$, colorectal cancer $(9 \%)$, and neuroendocrine cancer $(9 \%) ; 33 \%$ of the patients were staged T2 at baseline, $21 \% \mathrm{~T} 3$, and $17 \% \mathrm{~T} 4$. Overall, $47 \%$ of the patients had M1 stage disease and $43 \%$ M0. Of the 58 treated patients, $95 \%$ had received previous chemotherapy alone or chemotherapy in combination with surgery and/ or radiotherapy. Overall, $33 \%$ of patients received $\geq 4$ lines of chemotherapy. The most prior common chemotherapy regimens included fluorouracil $(40 \%)$, cisplatin $(33 \%)$, cyclophosphamide (29\%), docetaxel (28\%), and epirubicin $(22 \%)$. No patient had received surgery or radiotherapy alone, and only 1 patient $(2 \%)$ had received a combination of surgery and radiotherapy. Two patients (3\%) had received no previous therapy for disease control.

In the supradiaphragmatic group, the common sites of irradiation included lung/mediastinum $(n=13$ patients) and cerebral $(\mathrm{n}=10)$, followed by bone lesions ( $\mathrm{n}=7$ including 2 scapular, 2 vertebral, 2 sternum, and 1 rib lesion), other thoracic soft tissue $(\mathrm{n}=4)$, and cervical $(\mathrm{n}=3)$. In the subdiaphragmatic group, the common sites of irradiation were bone lesions ( $\mathrm{n}=12$ including 6 vertebral, 2 femoral, and 4 iliac lesions), followed by other soft tissue ( $\mathrm{n}=4$ including 1 abdomen, 1 hip, 1 suprarenal, and 1 thigh lesion), pelvis $(\mathrm{n}=4)$, and finally organ lesions $(n=2)$ and sites of adenopathy $(n=3)$.

At a median follow-up of 16 weeks (range, 0.6, $52.6), 83 \%$ of all treated patients completed the study. Of the 58 treated patients, 10 patients (17\%) discontinued treatment. The primary reason for discontinuation was AEs in 6 patients $(10 \%)$, progressive disease in 2 patients $(3 \%)$, protocol deviation in 1 patient $(2 \%)$, and nonmedical reason in 1 patient (2\%). No patient was lost to follow-up (Table 2).

\section{Efficacy}

Of the 51 patients evaluable for response, best overall response was $8 \%$ (1 CR, 3 PRs), and best locoregional response was $12 \%$ ( $1 \mathrm{CR}$ and $5 \mathrm{PRs})$ at a median 
Table 1: Baseline characteristics by schedule 1 and 2

\begin{tabular}{|c|c|c|c|}
\hline Characteristic & Schedule $1(n=33)$ & Schedule $2(n=25)$ & $\begin{array}{c}\text { Schedule } 1+\text { Schedule } 2 \\
(\mathrm{~N}=58)\end{array}$ \\
\hline Median age (range), years & $61(20-76)$ & $61(37-82)$ & $61.5(20-82)$ \\
\hline$>65$ years, $\mathrm{n}(\%)$ & $13(39 \%)$ & $9(36 \%)$ & $22(38 \%)$ \\
\hline Males, n (\%) & $13(39 \%)$ & $8(32 \%)$ & $21(36 \%)$ \\
\hline \multicolumn{4}{|l|}{$\begin{array}{l}\text { Eastern Cooperative Oncology } \\
\text { Group performance status, n (\%) }\end{array}$} \\
\hline Normal activity without restriction & $17(52 \%)$ & $6(24 \%)$ & $23(40 \%)$ \\
\hline $\begin{array}{l}\text { Ambulatory but restricted in } \\
\text { physical activity }\end{array}$ & $15(46 \%)$ & $14(56 \%)$ & $29(50 \%)$ \\
\hline $\begin{array}{l}\text { Ambulatory but unable to carry } \\
\text { out any work }\end{array}$ & $1(3 \%)$ & $5(20 \%)$ & $6(10 \%)$ \\
\hline Limited self-care, $50 \%$ in bed & 0 & 0 & 0 \\
\hline $\begin{array}{l}\text { Completely disabled, self-care } \\
\text { limited }\end{array}$ & 0 & 0 & 0 \\
\hline \multicolumn{4}{|l|}{ Types of prior therapies, $\mathrm{n}(\%)$} \\
\hline Antineoplastic agents & $30(91 \%)$ & $22(88 \%)$ & $52(90 \%)$ \\
\hline Endocrine therapy & $7(21 \%)$ & $7(28 \%)$ & $14(24 \%)$ \\
\hline All other therapeutic products & $9(27 \%)$ & $4(16 \%)$ & $13(22 \%)$ \\
\hline $\begin{array}{l}\text { Drugs for treatment of bone } \\
\text { diseases }\end{array}$ & $0(0 \%)$ & $3(12 \%)$ & $3(5 \%)$ \\
\hline $\begin{array}{l}\text { Pituitary and hypothalamic } \\
\text { hormones and analogues }\end{array}$ & $1(3 \%)$ & $1(4 \%)$ & $2(3 \%)$ \\
\hline Antiemetics and antinauseants & $1(3 \%)$ & $0(0 \%)$ & $1(2 \%)$ \\
\hline Diagnostic radiopharmaceuticals & $1(3 \%)$ & $0(0 \%)$ & $1(2 \%)$ \\
\hline Immunostimulants & $1(3 \%)$ & $0(0 \%)$ & $1(2 \%)$ \\
\hline $\begin{array}{l}\text { Sex hormones and modulators of } \\
\text { the genital system }\end{array}$ & $1(3 \%)$ & $0(0 \%)$ & $1(2 \%)$ \\
\hline \multicolumn{4}{|c|}{ Number of prior chemotherapy lines, $\mathrm{n}(\%)$} \\
\hline 1 & $6(18 \%)$ & $3(12 \%)$ & $9(16 \%)$ \\
\hline 2 & $7(21 \%)$ & $7(28 \%)$ & $14(24 \%)$ \\
\hline 3 & $9(27 \%)$ & $4(16 \%)$ & $13(22 \%)$ \\
\hline$\geq 4$ & $11(33 \%)$ & $8(32 \%)$ & $19(33 \%)$ \\
\hline $\begin{array}{l}\text { Median number of prior therapy } \\
\text { lines (range) }\end{array}$ & $3(1-10)$ & $3(1-8)$ & $3(1-10)$ \\
\hline \multicolumn{4}{|l|}{ Primary diagnosis, $\mathrm{n}(\%)$} \\
\hline Breast cancer & $10(30 \%)$ & $11(44 \%)$ & $21(36 \%)$ \\
\hline Lung cancer & $7(21 \%)$ & $7(28 \%)$ & $14(24 \%)$ \\
\hline Colorectal cancer & $5(15)$ & $0(0 \%)$ & $5(9 \%)$ \\
\hline Neuroendocrine cancer & $3(9 \%)$ & $2(8 \%)$ & $5(9 \%)$ \\
\hline Malignant melanoma & $3(9 \%)$ & $0(0 \%)$ & $3(5 \%)$ \\
\hline Other cancers & $5(15 \%)$ & $5(20 \%)$ & $10(16 \%)$ \\
\hline
\end{tabular}


Table 2: Patient disposition by schedule

\begin{tabular}{lccc}
\hline All Treated, $\mathbf{n}(\mathbf{\%})$ & Schedule $\mathbf{1}(\mathbf{n}=\mathbf{3 3})$ & Schedule 2 (n=25) & $\begin{array}{c}\text { Schedule 1 + Schedule 2 } \\
(\mathbf{N}=\mathbf{5 8})\end{array}$ \\
\hline Completed & $28(85 \%)$ & $20(80 \%)$ & $48(83 \%)$ \\
Withdrawn due to & $5(15 \%)$ & $5(20 \%)$ & $10(17 \%)$ \\
Adverse event & $4(12 \%)$ & $2(8 \%)$ & $6(10 \%)$ \\
Protocol deviation & $1(3 \%)$ & $1(4 \%)$ & $2(3 \%)$ \\
Progressive disease & $0(0 \%)$ & $1(4 \%)$ & $1(2 \%)$ \\
Nonmedical reason & $0(0 \%)$ & $1(4 \%)$ & $1(2 \%)$ \\
Lost to follow-up & $0(0 \%)$ & $0(0 \%)$ & $0(0 \%)$ \\
\hline
\end{tabular}

Table 3: Best overall and loco-regional response by schedule

\begin{tabular}{lccc}
\hline Best ORR, $\mathbf{( \% )}$ & Schedule 1 (n=30) & Schedule 2 (n=21) & $\begin{array}{c}\text { Schedule 1 + Schedule 2 } \\
\text { (N=51) }\end{array}$ \\
\hline CR & $0(0.0 \%)$ & $1(5 \%)$ & $1(2 \%)$ \\
PR & $1(3 \%)$ & $2(10 \%)$ & $3(6 \%)$ \\
SD & $17(57 \%)$ & $10(48 \%)$ & $27(53 \%)$ \\
PD & $10(33 \%)$ & $8(38 \%)$ & $18(35 \%)$ \\
Non-CR/Non-PD & $2(7 \%)$ & $0(0 \%)$ & $2(4 \%)$ \\
Objective response rate & $1(3 \%)$ & $3(14 \%)$ & $4(8 \%)$ \\
$95 \%$ CI & $0.1-17.2$ & $3.0-36.3$ & $2.2-18.9$ \\
\hline Best Loco-regional & Schedule 1(n=30) & Schedule 2(n=21) & Schedule 1+ Schedule \\
Response Rate, $\mathbf{n}(\mathbf{\%})$ & $0(0 \%)$ & $1(5 \%)$ & $\mathbf{2 ( N = 5 1 )}$ \\
\hline CR & $1(3 \%)$ & $4(19 \%)$ & $1(2 \%)$ \\
PR & $21(70 \%)$ & $11(52 \%)$ & $5(10 \%)$ \\
SD & $4(13 \%)$ & $4(19 \%)$ & $32(63 \%)$ \\
PD & $1(3 \%)$ & $1(5 \%)$ & $8(16 \%)$ \\
Non-CR/Non-PD & $1(3 \%)$ & $5(24 \%)$ & $2(4 \%)$ \\
Objective response rate & $0.1-17.2$ & $8.2-47.2$ & $6(12 \%)$ \\
$95 \%$ CI & & & $4.4-23.9$ \\
\hline
\end{tabular}

follow-up of 16 weeks (Table 3). The best overall and loco-regional response by supra- and subdiaphragmatic group is shown in Supplementary Table 2.

Ten patients had brain lesions as target or non-target lesions, with the most common primary tumor being breast adenocarcinoma $(n=6)$, followed by 1 case each of adenocarcinoma of the colon, adenocarcinoma of the esophagus, adenocarcinoma of the lung, and melanoma. Loco-regional efficacy assessment was performed in 7 patients. Of 4 patients with brain lesions as target lesions, 1 patient achieved a loco-regional best response of PR, and 3 achieved stable disease (loco-regional response rate $25 \%$ ). Of 6 patients with brain lesions recorded as nontarget lesions, the loco-regional best responses were 1 $\mathrm{CR}, 4$ non-CR/non-PD, and $1 \mathrm{PD}$ (loco-regional response rate $17 \%$ ). The primary tumor histology for the patient achieving a CR was lung adenocarcinoma. Three patients did not complete the tumor evaluation visit at week 6 as per protocol requirement (they did complete the followup visit) and were therefore considered non-evaluable for final efficacy assessment.

Safety

\section{MTDs and DLTs for schedule 1 and 2}

Abexinostat was administered BID from day 1 to 5 and during radiotherapy from day 8 to 12 and 15 to 19 . This schedule of 5 days on/2 days off (schedule 1) chosen for this study was used with a starting daily dose of $15 \mathrm{mg} / \mathrm{m}^{2}$ BID.

The schedule of 4 days on 3 days off (schedule 2) was based on tolerability of abexinostat given as standalone therapy observed in the clinical study CL1-78454002 [18], a study performed during the same timeframe. This schedule is associated with the smallest platelet 
decrease [18], and dose levels $75 \mathrm{mg} / \mathrm{m}^{2}$ BID, $90 \mathrm{mg} / \mathrm{m}^{2}$ $\mathrm{BID}$, and $105 \mathrm{mg} / \mathrm{m}^{2}$ BID. Changes in the abexinostat administration schedule were made to increase the hematological therapeutic window of abexinostat.

Twenty-seven patients were evaluated for DLT in schedule 1. MTD1 for schedule 1 was reached at $60 \mathrm{mg} / \mathrm{m}^{2}$ in the supradiaphragmatic group (2 DLTs for 6 evaluable patients), after which dose escalation was initiated from MTD1 (60 mg/m² BID) with schedule 2 of administration (i.e., 4 days on/3 days off per week over 2 weeks). MTD1 of schedule 1 was not reached at $60 \mathrm{mg} / \mathrm{m}^{2}$ BID in the subdiaphragmatic group, after which dose escalation started from the next dose level $\left(75 \mathrm{mg} / \mathrm{m}^{2}\right.$ BID) with schedule 2 .

Twenty-two patients were evaluated for DLT in schedule 2. MTD2 of schedule 2 was reached at 105 $\mathrm{mg} / \mathrm{m}^{2}(160 \mathrm{mg})$ for the subdiaphragmatic group. Four patients treated at the dose level of $105 \mathrm{mg} / \mathrm{m}^{2}(160 \mathrm{mg})$ across supra/subdiaphragmatic groups were evaluable for assessment of DLTs. Numbers of patients and DLTs at each dose level within the supra- and subdiaphragmatic groups are given in Supplementary Table 3.

At the highest dose level of $105 \mathrm{mg} / \mathrm{m}^{2}$ (160 $\mathrm{mg}$ ), 3 out of 4 evaluable patients had a DLT (2 in subdiaphragmatic group and 1 in supradiaphragmatic group) of hematologic or gastrointestinal origin (2 cases of grade 4 thrombocytopenia and 1 case of grade 3 diarrhea). No clinically meaningful differences were observed between the supra- and subdiaphragmatic groups in terms of frequencies of DLTs. Based on these DLT observations, the study sponsor and study investigators determined that MTD2 was reached at $105 \mathrm{mg} / \mathrm{m}^{2}(160$ $\mathrm{mg}$ ), and the recommended phase 2 dose was therefore considered $90 \mathrm{mg} / \mathrm{m}^{2}$ (140 mg) for both subdiaphragmatic and supradiaphragmatic groups.

Overall, 98\% of all treated patients experienced 1 or more AE. Grade $\geq 3$ AEs occurred in $55 \%$ of patients. Grade $\geq 3$ hematologic AEs occurring in more than 1 patient included thrombocytopenia ( $\mathrm{n}=10,17 \%)$, lymphopenia $(n=7,12 \%)$, and neutropenia $(n=2,3 \%)$ (Table 4). Grade $\geq 3$ nonhematologic AEs occurring in more than 1 patient included hypokalemia $(n=4$, $7 \%)$, asthenia $(n=3,5 \%)$, diarrhea $(n=2,3 \%)$, gammaglutamyltransferase increased $(n=2,3 \%)$, and decreased appetite $(n=2,3 \%)$ (Table 4$)$. No substantial differences were observed in the frequencies of AEs between the supra- and subdiaphragmatic groups. Seventeen patients (29\%) experienced an SAE, which were grade $\geq 3$ in $22 \%$ of patients. Treatment-related SAEs occurred in $12 \%$ of patients ( $9 \%$ grade $\geq 3$ ). Malignant neoplasm progression leading to death was reported in 3 patients $(5 \%)$.

Six patients $(10 \%)$ discontinued treatment due to AEs ( 4 due to thrombocytopenia, 1 due to vomiting and diarrhea, 1 due to cholangiolitis). No grade $\geq 3$ prolongation of the QTc interval or grade $\geq 2$ prolongation of the QTc interval persisting more than 14 days (as determined by a central electrocardiogram [ECG] reading center) was observed, and no patient discontinued due to QTc interval prolongation.

\section{DISCUSSION}

Current treatments for patients with advanced metastatic solid tumors tend to be only palliative, which represents an area of unmet need. Concomitant chemoradiotherapy is an established treatment modality for solid tumors, particularly for loco-regional disease control [19]; however, the main drawback of chemotherapy when combined with radiotherapy is the possible amplification of radiation-induced acute and late toxicity to normal tissues. Thus, there is a need to optimize radiotherapy of metastatic solid tumors using combination agents that are active and tolerable.

Through preclinical studies in cancer models, HDACIs are emerging as radiation sensitizers, suggesting their clinical potential in combination with radiotherapy. Our preclinical data using an EMT-6 tumor-bearing murine model demonstrated that combination of lowdose radiation ( $2 \mathrm{~Gy}$ ) and abexinostat led to inhibition of tumor growth and improved survival benefit compared with abexinostat alone or low-dose radiation alone (Supplementary Figures 1A and 1B). Previous studies demonstrated that following IV administration of radiolabeled $\left[{ }^{14} \mathrm{C}\right]$-abexinostat, unchanged parent drug was the major component in the plasma, and radioactivity was widely distributed in all tissues, especially cerebellum, cerebrum, and cerebrospinal fluid, indicating that radiolabeled abexinostat crossed the blood-brain barrier (data not shown).

The current phase 1 study was designed to evaluate initial safety and tolerability of the novel HDACI abexinostat in combination with radiotherapy, with the intent of systemic disease assessment, such as secondary tumor sites in the setting of palliation, where long-term toxicities are different from those in early-stage localized disease [20]. The major limitation of the use of a trial design, such as ours, focusing on patients receiving radiation with a palliative intent (i.e., $30 \mathrm{~Gy}$ in 10 fractions of $3 \mathrm{~Gy}$ ) without focusing on a specifically defined tumor location is that the trial generates information about normal tissue tolerance (the major objective of a radiation + new drug phase 1 trial), but only after irradiation of variable normal tissues and dose-limiting organs. Given the fact that the normal lung and bowel are major dose-limiting organs, but display different volume and radiation dose tolerance, the design of our trial included 2 distinct cohorts, the subdiaphragmatic group that is more likely to accrue patients for whom radiation fields encompass the bowel, and the supradiaphragmatic group that is more likely to include the lung into the irradiated volume [20]. However, we believe that this type of 
Table 4: Overview of common AEs $(>10 \%)$ and grade $\geq 3$ AEs in $>1$ patient

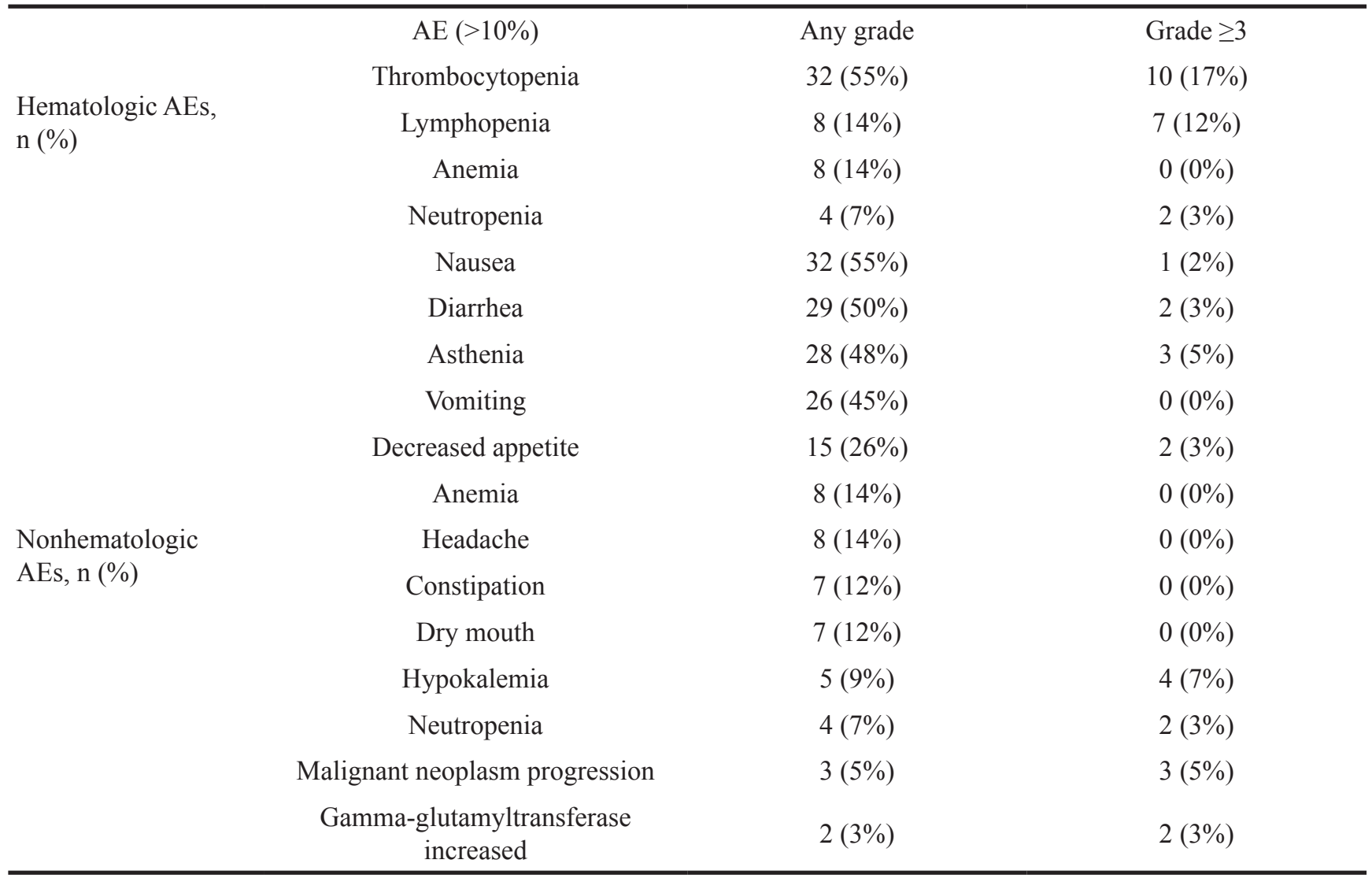

design was probably the safest in terms of risk/benefit balance when a drug is combined with radiation for the first time in humans. Previously used by other groups $[21,22]$, this type of design offers the advantage of a better safety balance when a drug is combined with radiation in human subjects for the first time. This is especially important because this type of trials select patients requiring palliative radiotherapy without concurrent chemotherapy (platinum, 5-fluorouracil, etc). On the contrary, a trial addressing solely one type of localized cancer treated with radiotherapy in a curative intent might have provided more information in terms of tolerance for a given organ; however, this type of trial would have inevitability carried the risk of major, permanent toxicity in a population of patients amenable to cure.

$\mathrm{PK} /$ pharmacodynamic modeling studies have been able to predict the optimal schedule of abexinostat allowing higher doses with minimal thrombocytopenia, showing that an abexinostat dosing schedule of 4 days on/3 days off (schedule 2 in the present study) was associated with smaller platelet decrease. The MTD2 reached for this optimized schedule was $105 \mathrm{mg} / \mathrm{m}^{2} \mathrm{BID}$, and the recommended dose, $90 \mathrm{mg} / \mathrm{m}^{2}$ BID [18]. These doses are in agreement with the findings of this study. We speculated that the unique PK profile of abexinostat (halflife 4-5 hours) with twice daily dosing [18] may provide an advantageous safety profile in combination with radiotherapy for the treatment of advanced solid tumors.

Safety data demonstrated good tolerability of abexinostat combined with hypofractionated palliative radiotherapy in this patient population of advanced solid tumors. No marked differences were observed in the frequencies of DLTs or AEs between the supraand subdiaphragmatic groups. Thrombocytopenia was the most common hematologic toxicity, reported in $55 \%$ of the patients, with grade $\geq 3$ in $17 \%$. This result is consistent with the finding that thrombocytopenia is less frequently observed with the 4 days on $/ 3$ days off schedule [20] compared with a high frequency of grade $\geq 3$ thrombocytopenia with a 14-day dosing schedule (21-day cycle) in patients with non-Hodgkin lymphoma and chronic lymphocytic leukemia [21]. The most common nonhematologic toxicities reported were nausea, diarrhea, asthenia, and vomiting, which have been commonly observed in previous studies with HDACIs. Cardiotoxicities such as QT/QTc prolongation have been a safety concern with the use of existing HDACIs in some instances $[23,24]$. In the current study, no grade $\geq 3$ prolongation of the QTc interval (as determined by a central ECG reading center) was observed. Only 2 patients on schedule 2 had a grade $1 / 2$ prolongation of QTc, and no patient discontinued treatment due to QTc interval prolongation. QT prolongations (grade 2) in both patients 
resolved without dose reductions, were not serious, and were assessed by the investigator as unlikely related to the study treatment. Overall, only $10 \%$ of all treated patients discontinued treatment, demonstrating a tolerable safety profile of abexinostat in combination with radiotherapy.

The combination of abexinostat and low-dose radiotherapy led to a loco-regional response rate of $12 \%$, with 5 PRs and $1 \mathrm{CR}$ in the current study, and a substantial proportion of patients $(63 \%)$ achieved a best loco-regional response of stable disease. A longer follow-up and a larger sample size is warranted to monitor these responses. No clinically significant differences in efficacy were observed between schedules 1 and 2 or supra/subdiaphragmatic groups. Due to the larger number of patients enrolled in some dose cohorts, a statistically valid conclusion could not be made for the dose/response relationship.

The ability of abexinostat to cross the blood-brain barrier demonstrated in pre-clinical studies is supported by the encouraging loco-regional responses observed in patients with brain lesions in this study and suggests that abexinostat in combination with radiotherapy may constitute a treatment option for these lesions. These findings are notable (particularly a best response of CR in 1 patient with brain lesions as non-target lesion) given the modest activity of currently approved HDACIs, vorinostat and romidepsin, as single agents or in combination therapy in patients with high-grade gliomas [25] and with glioblastoma [26-28].

Of note, HDACIs have been demonstrated to exert immunomodulatory effects in cancer cells, such as enhanced expression of natural killer cell-activating ligands, MHC class I and II molecules, and CD40 molecules [29-31]. It was demonstrated that vorinostat elicits antitumor immunity in colon cancer mouse models through immunogenic cell death [32]. The abscopal effect of radiation has been shown to be immune-mediated [33], in part by IL-12 [34], dendritic cells, and T cells [35]. Thus, the immunomodulatory effects of abexinostat have marked implications for radiotherapy and warrant evaluation of the combination in future radiotherapy studies.Subsequent trials with a focus on a particular tumor type are needed that can first test the combination in a phase I/II setting, especially if higher radiation doses or concurrent chemotherapy are investigated in combination with abexinostat.

In summary, oral abexinostat in combination with hypofractionated radiotherapy demonstrates activity with specific loco-regional responses in patients with metastatic solid tumors who have undergone prior surgery with

\section{A. Schedule 1}

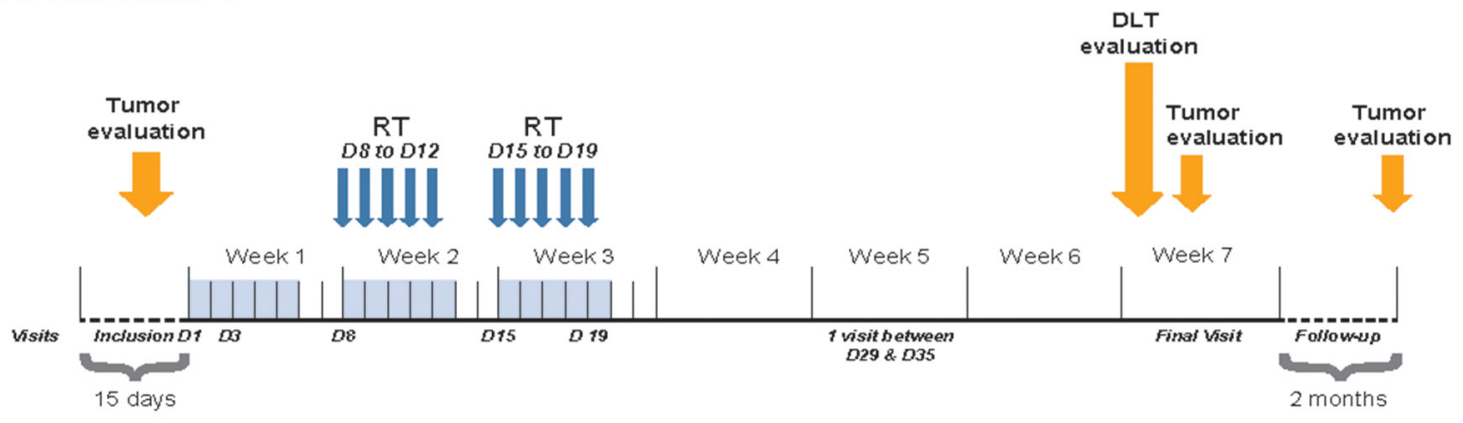

RT, radiotherapy ( 3 Grays per day for 5 consecutive days for 2 weeks)

Abx BID for 5 consecutive days for the 3 weeks (from week 1 to week 3 )

\section{B. Schedule 2}

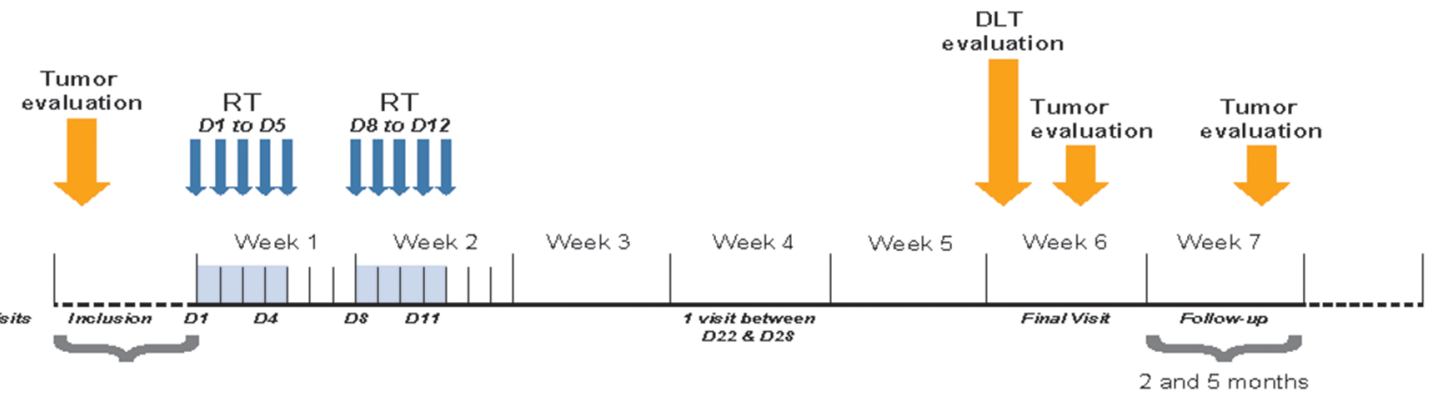

RT, radiotherapy ( 3 Grays per day for 5 consecutive days for 2 weeks)

Abx BID for 4 consecutive days for 2 weeks (from week 1 to week 2)

Figure 1: Study Design. A. Schedule 1. B. Schedule 2. 
chemotherapy or chemoradiotherapy. The combination is well tolerated, with the majority of AEs being grade 1/2. Although our patient population was small, based on the positive results observed, the potential of this combination for the treatment of patients with brain lesions should be investigated further in a larger population and with longer follow-up.

\section{MATERIALS AND METHODS}

\section{Ethics statement}

Investigation has been conducted in accordance with the ethical standards and according to the Declaration of Helsinki and according to national and international guidelines and has been approved by the authors' institutional review board. Written consent was obtained from each patient prior to study entry.

\section{EMT-6 murine breast carcinoma model}

Tumor volumes before and after treatment was assessed in 4 groups: vehicle only, abexinostat only, lowradiation only, and combination of low-dose radiotherapy and abexinostat ( $\mathrm{n}=10$ each). Female BALB/c mice 8 to 12 weeks old were injected in the flank region with $5 \times 10^{6}$ EMT-6 tumor cells in 0\% Matrigel (cell injection volume was $0.1 \mathrm{~mL} / \mathrm{mouse}$ ). Treatment was started on matched pairs when tumors reached an average size of 50-70 $\mathrm{mm}^{3}$. Tumors were irradiated at a dose of 2 Gy/animal for 5 days ( 1 cycle). Abexinostat (IP) was administered concurrently at a dose of $12 \mathrm{mg} / \mathrm{kg}$ BID, 6 hours apart, 5 days on/2 days off/week for 4 weeks. Mice were monitored until tumor volume reached $2000 \mathrm{~mm}^{3}$ or 45 days, whichever came first. Tumor volumes were measured twice per week in two dimensions using a calipers, and the volume was expressed in $\mathrm{mm}^{3}$ using the formula: $\mathrm{V}=0.5 a \times b^{2}$ where $a$ and $b$ are the long and short diameters of the tumor, respectively. Kaplan-Meier survival curves of EMT-6 tumor-bearing mice were analyzed until 45 days after the start of treatment.

\section{Study design}

We conducted a multicenter, open-label, exploratory phase 1, dose-escalation study at 6 centers in Italy and France. Eligible patients had histologically confirmed solid tumors with measurable disease and required a course of hypofractionated palliative radiotherapy. These included patients with metastatic tumors with locally advanced disease for which primary tumor management before systemic treatment was required; primary tumors in relapse, tumors not previously irradiated, and tumors requiring hypofractionated radiotherapy; metastatic localization requiring hypofractionated radiotherapy; and patients not fit for conventional chemoradiotherapy. Other eligibility criteria included an Eastern Cooperative
Oncology Group performance status $\leq 2$; an estimated life expectancy $>20$ weeks; and adequate hematologic, cardiac, renal, and hepatic functions. Exclusion criteria included allogenic bone marrow transplant; major surgery within 4 weeks before first day of treatment; chemotherapy within 3 weeks (6 weeks in case of nitrosoureas) of study treatment administration; previous radiotherapy on the same area; immunotherapy or hormone therapy within 2 weeks except stable luteinizing hormone-releasing hormone agonist therapy for prostate cancer, stable oral glucocorticoid and mineralocorticoid replacement for adrenal insufficiency, stable mitotane for adrenal carcinoma, or oral contraceptives treatment with other antineoplastic agents within 3 weeks; concurrent therapeutic anticoagulation by vitamin $\mathrm{K}$ antagonists; any treatment known to prolong QTc interval; treatment with valproic acid within 5 days of study drug administration; and prophylactic use of growth factors.

\section{Abexinostat and radiotherapy schedule}

Abexinostat in combination with a fixed radiation dose (30 Gy in 10 daily fractions of 3 Gy, 5 days per week over 2 weeks) was administered in 2 schedules. For schedule 1, treatment with abexinostat started 1 week before starting hypofractionated radiotherapy in order to induce biological modifications in tumor cells before radiation and then continued concomitantly with hypofractionated radiotherapy for 2 weeks. Abexinostat was given twice a day (BID) from day 1 to 5 and during radiotherapy from day 8 to 12 and 15 to 19 . The schedule of 5 days on/ 2 days off (schedule 1) chosen for this study was based on tolerability and activity of abexinostat observed in the clinical study PCYC-0402 [18]. The choice of this schedule also allowed the administration of abexinostat concomitantly with radiotherapy. In order to increase safety margin of the combination of abexinostat with radiotherapy, we chose a starting daily dose of 15 $\mathrm{mg} / \mathrm{m}^{2}$ BID for the present study, which is $25 \%$ of the recommended dose of $60 \mathrm{mg} / \mathrm{m}^{2}$ BID found in PCYC0402 study and 1 dose level below the starting dose used in the PCYC-0402 study.

For schedule 2, abexinostat and hypofractionated radiotherapy were administered concomitantly from day 1 to day 4 and from day 8 to day 11 (Figure 1). The schedule of 4 days on/3 days off (schedule 2) chosen for this study was based on tolerability of abexinostat observed in the clinical study CL1-78454-002 [20]. This schedule is associated with the smallest platelet decrease [20], and dose levels $75 \mathrm{mg} / \mathrm{m}^{2}$ BID, $90 \mathrm{mg} / \mathrm{m}^{2}$ BID, and $105 \mathrm{mg} /$ $\mathrm{m}^{2}$ BID were investigated.

For both schedules, radiotherapy was administered using a 3-dimensional conformal technique. Patients received hypofractionated external beam irradiation using linear accelerators with photon energies $\geq 4$ megavolts. Patients within the 2 schedules were categorized 
according to the localization of the radiation field (supradiaphragmatic and subdiaphragmatic group) in order to evaluate radiotherapy-related potential toxicities specific to the irradiated regions.

Dose escalation followed a $3+3$ algorithm-based design and continued until maximum tolerated dose (MTD) was achieved based on protocol-defined doselimiting toxicities (DLTs) defined as the occurrence in cycle 1 of any of the following: a grade 3 nonhematologic adverse event (AE), grade 3 prolongation of the QTc interval, grade 4 neutropenia lasting $>5$ days on growth factors, grade 4 thrombocytopenia, or failure to restart abexinostat administration within 2 weeks. Dose escalation to the next level proceeded after DLT assessment over the 3 -week treatment period for schedule 1 or over the 2 -week treatment period for schedule 2, and the following 3 weeks without treatment. Tumor evaluation was performed 3 weeks after treatment completion and after a 2-month follow-up. AEs at each visit were graded according to the Common Toxicity Criteria for Adverse Events [CTCAE] v3.0) [36]. Serious AEs (SAEs) were those events that were fatal, life threatening, required hospitalization, disabling, or judged to be medically significant.

\section{Study objectives and assessments}

The primary objectives of the study were to assess the safety and tolerability of oral abexinostat given in combination with standard hypofractionated radiotherapy in patients with advanced solid tumor in terms of MTD and to establish the recommended phase 2 dose. The secondary objectives were to assess safety and tolerability of the combination, to evaluate tumor responses of the combination, and to analyze the pharmacokinetic (PK) profile of abexinostat and its metabolites. Tumor responses as defined by loco-regional response rate and overall response rate (ORR) were assessed per the revised Response Evaluation Criteria in Solid Tumors (RECIST version 1.1) [37].

ORR was defined as proportion of patients exhibiting a complete response (CR) or a partial response (PR) per the RECIST criteria (version 1.1). Loco-regional response rate was defined as the proportion of patients exhibiting a CR or a PR per the RECIST criteria (version 1.1) using tumor lesions encompassed within the radiation field. Lesions were evaluated by both clinical exam and computed tomography imaging. Electrocardiograms were performed at screening and both at pre-first dose and 1 to 1.5 hours post-first dose on days $1,3,8,15,19$, and final visit of schedule 1 and days $1,4,8,11$, and final visit of schedule 2 .

\section{Statistical analysis}

The efficacy analyses were performed in the full analysis set, which included patients who had received at least 1 dose of study treatment and who had at least
1 baseline and 1 post-baseline tumor evaluation. Best overall response per investigator assessment was provided for each dose level and overall population by schedule and group. Response rates (loco-regional response and ORR) and corresponding exact binomial 95\% confidence interval were provided. Safety analyses were conducted on the safety set in each anatomically based group by dose level. Safety was assessed with a description of DLTs on the DLT-evaluable population comprising patients who completed the total treatment period of 3 weeks for schedule 1 and 2 weeks for schedule 2 and 3 weeks without treatment period (i.e., evaluation period) following total treatment period or who discontinued due to DLT during total treatment or 3 weeks without treatment period. Descriptive statistics were used for evaluating toxicity (according to CTCAE v3.0), AEs, physical examination, performance status, vital signs, and laboratory tests.

\section{ACKNOWLEDGMENTS}

The authors would like to thank the patients who participated in the study and their supportive families, and the investigators and clinical research staff from the study centers. Medical writing assistance was provided by Swati Ghatpande, PhD, and was funded by Pharmacyclics LLC, an AbbVie company.

\section{CONFLICTS OF INTEREST}

ED, consulting or advisory role for Roche, research funding from Servier, Roche, AstraZeneca, travel accommodations from Merck, Roche; ECJM, consulting or advisory role for Merck; VG, consulting or advisory role for and travel accommodations from Pfizer, Novartis, Eli Lilly, Roche, AstraZeneca, Boehringer Ingelheim; PAZ, no relevant financial disclosures; JM, no relevant financial disclosures; JCS, no relevant financial disclosures; IK, patients, royalties, or other intellectual property for Servier; JH, employment with Pharmacyclics and Astellas Pharma (Agensys), stock or other ownership for AbbVie; YL, employment with Pharmacyclics, stock or other ownership for AbbVie; EL, employment for Pharmacyclics, Theorem Clinical Research, stock or other ownership for AbbVie; RV, employment for Pharmacyclics, stock or other ownership for AbbVie; TG, employment and patents, royalties, or other intellectual property for Pharmacyclics, stock or other ownership for AbbVie; SR, travel accommodations from Novartis.

\section{GRANT SUPPORT}

Not applicable. Trial was funded by Pharmacyclics LLC, an AbbVie Company. 


\section{REFERENCES}

1. Yoo CB, Jones PA. Epigenetic therapy of cancer: past, present and future. Nat Rev Drug Discov. 2006; 5:37-50.

2. Luo JY, Su F, Chen DL, Shiloh A, Gu W. Deacetylation of p53 modulates its effect on cell growth and apoptosis. Nature. 2000; 408:377-381.

3. Duan H, Heckman CA, Boxer LM. Histone deacetylase inhibitors down-regulate bcl-2 expression and induce apoptosis in $\mathrm{t}(14 ; 18)$ lymphomas. Mol Cell Biol. 2005; 25:1608-1619.

4. Doi T, Hamaguchi T, Shirao K, Chin K, Hatake K, Noguchi K, Otsuki T, Mehta A, Ohtsu A. Evaluation of safety, pharmacokinetics, and efficacy of vorinostat, a histone deacetylase inhibitor, in the treatment of gastrointestinal (gi) cancer in a phase I clinical trial. Int J Clin Oncol. 2013; 18:87-95.

5. Amiri-Kordestani L, Luchenko V, Peer CJ, Ghafourian K, Reynolds J, Draper D, Frye R, Woo S, Venzon D, Wright J, Skarulis M, Figg WE, Fojo T, et al. Phase I trial of a new schedule of romidepsin in patients with advanced cancers. Clin Cancer Res. 2013; 19:4499-4507.

6. Ramalingam SS, Belani CP, Ruel C, Frankel P, Gititz B, Kocywas M, Espinoza-Delgado I, Gandara D. Phase II study of belinostat (PXD101), a histone deacetylase inhibitor, for second line therapy of advanced malignant pleural mesothelioma. J Thorac Oncol. 2009; 4:97-101.

7. Woyach JA, Kloos RT, Ringel MD, Arbogast D, Collamore M, Zwiebel JA, Grever M, Villalona-Calero M, Shah MH. Lack of therapeutic effect of the histone deacetylase inhibitor vorinostat in patients with metastatic radioiodinerefractory thyroid carcinoma. J Clin Endocrinol Metab. 2009; 94:164-170.

8. Martinet N, Bertrand P. Interpreting clinical assays for histone deacetylase inhibitors. Cancer Manage Res. 2011; 3:117-141

9. Whitehead RP, Rankin C, Hoff PM, Gold PJ, Billingsley KG, Chapman RA, Wong L, Ward JH, Abbruzzese JL, Blanke CD. Phase 2 trial of romidepsin (NSC-630176) in previously treated colorectal cancer patients with advanced disease: a Southwest Oncology Group study (S0336). Invest New Drugs. 2009; 27:469-475.

10. Stadler WM, Margolin K, Ferber S, McCulloch W, Thompson JA. A Phase 2 study of depsipeptide in refractory metastatic renal cell cancer. Clin Genitourin Cancer. 2006; 5:57-60.

11. Giaccone G, Rajan A, Berman A, Kelly RJ, Szabo E, LopezChavez A, Trepel J, Lee MJ, Cao L, Espinoza-Delgado I, Spittler J, Loehrer PJ Sr. Phase II study of belinostat in patients with recurrent or refractory advanced thymic epithelial tumors. J Clin Oncol. 2011; 29:2052-2059.

12. Sholler GS, Currier EA, Dutta A, Slavik MA, Illenye SA, Mendonca MC, Dragon J, Roberts SS, Bond JP. PCI24781 (abexinostat), a novel histone deacetylase inhibitor, induces reactive oxygen species-dependent apoptosis and is synergistic with bortezomib in neuroblastoma. J Cancer Ther Res. 2013; 2:21.

13. Lopez G, Liu J, Ren W, Wei W, Wang S, Lahat G, Zhu QS, Bornmann WG, McConkey DJ, Pollock RE, Lev DC. Combining PCI-24781, a novel histone deacetylase inhibitor, with chemotherapy for the treatment of soft tissue sarcoma. Clin Cancer Res. 2009; 15:3472-3483.

14. Rivera-Del Valle N, Gao S, Miller CP, Fulbright J, Gonzales C, Sirisawad M, Steggerda S, Wheler J, Balasubramanian S, Chandra J. PCI-24781, a novel hydroxamic acid HDAC inhibitor, exerts cytotoxicity and histone alterations via caspase- 8 and FADD in leukemia cells. Int J Cell Biol. 2010; 2010:207420.

15. Mottamal M, Zheng S, Huang TL, Wang G. Histone deacetylase inhibitors in clinical studies as templates for new anticancer agents. Molecules. 2015; 20:3898-3941.

16. Banuelos CA, Banáth JP, MacPhail SH, Zhao J, Reitsema T, Olive PL. Radiosensitization by the histone deacetylase inhibitor PCI-24781. Clin Cancer Res. 2007; 13:6816-6826.

17. Adimoolan S, Sirisawad M, Chen J, Thiemann P, Ford JM, Buggy JJ. HDAC inhibitor PCI-24781 decreases RAD51 expression and inhibits homologous recombination. Proc Natl Acad Sci USA. 2007; 104:19482-19487.

18. Fouliard S, Robert R, Jacquet-Bescond A, du Rieu QC, Balasubramanian S, Loury D, Loriot Y, Hollebecque A, Kloos I, Soria JC, Chenel M, Depil S. Pharmacokinetic/ pharmacodynamic modelling-based optimisation of administration schedule for the histone deacetylase inhibitor abexinostat (S78454/PCI-24781) in phase I. Eur J Cancer. 2013; 49:2791-2797.

19. Seiwert TY, Salama JK, Vokes EE. The concurrent chemoradiation paradigm--general principles. Nat Clin Pract Oncol. 2007; 4:86-100.

20. Deutsch E, Soria JC, Armand JP. New concepts for phase I trials: evaluating new drugs combined with radiation therapy. Nat Clin Pract Oncol. 2005; 2:456-465.

21. Harrington KJ, Hingorani M, Tanay MA, Hickey J, Bhide SA, Clarke PM, Renouf LC, Thway K, Sibtain A, McNeish IA, Newbold KL, Goldsweig H, Coffin R, et al. Phase I/ II study of oncolytic HSV GM-CSF in combination with radiotherapy and cisplatin in untreated stage III/IV squamous cell cancer of the head and neck. Clin Cancer Res. 2010; 16:4005-4015.

22. Pugh TJ, Chen C, Rabinovitch R, Eckhardt SG, Rusthoven KE, Swing R, Raben D. Phase I trial of bortezomib and concurrent external beam radiation in patients with advanced solid malignancies. Int J Radiat Oncol Biol Phys. 2010; 78:521-526.

23. Ribrag V, Kim WS, Bouabdallah R, Lim ST, Coiffier B, Felloussi Z, Kloos I, Luan Y, Graef Y, Morschhauser F. Safety and efficacy of abexinostat, a pan- histone deacetylase inhibitor, in non-Hodgkin lymphoma and 
chronic lymphocytic leukemia: results of an ongoing phase 2 study. Blood. 2015; 126:256.

24. Gryder BE, Sodji QH, Oyelere AK. Targeted cancer therapy: giving histone deacetylase inhibitors all they need to succeed. Future Med Chem. 2012; 4:505-524.

25. Lee EQ, Puduvalli VK, Reid JM, Kuhn JG, Lamborn KR, Cloughesy TF, Chang SM, Drappatz J, Yung WK, Gilbert MR, Robins HI, Lieberman FS, Lassman AB, et al. Phase I study of vorinostat in combination with temozolomide in patients with high-grade gliomas: North American Brain Tumor Consortium Study 04-03. Clin Cancer Res. 2012; 18:6032-6039.

26. Galanis E, Jaeckle KA, Maurer MJ, Reid JM, Ames MM, Hardwick JS, Reilly JF, Loboda A, Nebozhyn M, Fantin VR, Richon VM, Scheithauer B, Giannini C, et al. Phase II trial of vorinostat in recurrent glioblastoma multiforme: a north central cancer treatment group study. J Clin Oncol. 2009; 27:2052-2058.

27. Iwamoto FM, Lamborn KR, Kuhn JG, Wen PY, Yung WK, Gilbert MR, Chang SM, Lieberman FS, Prados MD, Fine HA. A phase I/II trial of the histone deacetylases inhibitor romidepsin for adults with recurrent malignant glioma: North American Brain Tumor Consortium Study 03-03. Neuro Oncol. 2011; 13:509-516.

28. Friday BB, Anderson SK, Buckner J, Yu C, Giannini C, Geoffroy F, Schwerkoske J, Mazurczak M, Gross H, Pajon E, Jaeckle K, Galanis E. Phase II trial of vorinostat in combination with bortezomib in recurrent glioblastoma: a North Central Cancer Treatment Group study. Neuro Oncol. 2012; 14:215-221.

29. Khan AN, Magner WJ, Tomasi TB. An epigenetic vaccine model active in the prevention and treatment of melanoma. J Transl Med. 2007; 5:64.

30. Magner WJ, Kazim AL, Stewart C, Romano MA, Catalano G, Grande C, Keiser N, Santaiello F, Tomasi TB. Activation of MHC class I, II, and CD40 gene expression by histone deacetylase inhibitors. J Immunol. 2000; 165:7017-7024.

31. Skov S, Pedersen MT, Andresen L, Straten PT, Woetmann A, Odum N. Cancer cells become susceptible to natural killer cell killing after exposure to histone deacetylase inhibitors due to glycogen synthase kinase-3-dependent expression of MHC class I-related chain A and B. Cancer Res. 2005; 65:11136-11145.

32. West AC, Mattarollo SR, Shortt J, Cluse LA, Christiansen AJ, Smyth MJ, Johnstone RW. An intact immune system is required for the anticancer activities of histone deacetylase inhibitors. Cancer Res. 2013; 73:7265-7276.

33. Liauw SL, Connell PP, Weichselbaum RR. New paradigms and future challenges in radiation oncology: an update of biological targets and technology. Sci Transl Med. 2013; 5:173sr2.

34. Seetharam S, Staba MJ, Schumm LP, Schreiber K, Schreiber H, Kufe DW, Weichselbaum RR. Enhanced eradication of local and distant tumors by genetically produced interleukin-12 and radiation. Int J Oncol. 1999; 15:769-773.

35. Demaria S, Ng B, Devitt ML, Babb JS, Kawashima N, Liebes L, Formenti SC. Ionizing radiation inhibition of distant untreated tumors (abscopal effect) is immune mediated. Int J Radiat Oncol Biol Phys. 2004; 58:862-870.

36. National Cancer Institute Common Terminology Criteria for Adverse Events (CTCAE) v4.0. Available at: http://ctep. cancer.gov/protocolDevelopment/electronic_applications/ docs/ctcaev3.pdf.

37. Eisenhauer EA, Therasse P, Bogaerts J, Schwartz LH, Sargent D, Ford R, Dancey J, Arbuck S, Gwyther S, Mooney M, Rubinstein L, Shankar L, Dodd L, et al. New response evaluation criteria in solid tumours: Revised RECIST guideline (version 1.1). Eur J Cancer. 2009; 45:228-247. 